\title{
Staggered Numerical Modeling for Investigating the Effectiveness of Embankment and Vegetation on the Reduction of Wave Run-up
}

\author{
Iryanto $^{1}$, Muhamad Mustamiin ${ }^{2}$, Munengsih Sari Bunga ${ }^{3}$, Ikha Magdalena ${ }^{4}$, and Novry Erwina ${ }^{5}$ \\ \{iryanto.math@yahoo.com ${ }^{1}$, m.mustamiin@gmail.com ${ }^{2}$, nengslim85@gmail.com ${ }^{3}$ \} \\ Informatics Department, Politeknik Negeri Indramayu, Indonesia ${ }^{1}$, Industrial and Financial \\ Mathematics Research Group, Faculty of Mathematics and Natural Sciences, Institut Teknologi \\ Bandung, Indonesia ${ }^{2}$
}

\begin{abstract}
In this research, we develop a mathematical model to investigate the effectiveness of embankment and vegetation on the reduction of wave run-up. We solve the model numerically using a staggered conservative scheme. Wave run-up testing on a sloping beach is conducted to show the accuracy of our numerical scheme. In this case, we compare our numerical results with data of laboratory experiments of Synolakis provided in [9]. The results of the comparison show that the numerical results are in good agreement with the experimental data. Further, the results of the influence of the vegetation, embankment, and combination of both of them are presented in the article.
\end{abstract}

Keywords: Staggered numerical modeling, staggered conservative scheme, embankment, vegetation, wave run-up, shallow water model

\section{Introduction}

Sea wave has potential to threat (even to destroy) coastal area. Some examples of the phenomena are tsunami and abrasion. In order to minimize the destruction of the area, some methods are used such as by planting vegetation or by building embankment as a wave barrier. This article is focused on numerical modeling for investigating the effectiveness of embankment and vegetation on the reduction wave run-up.

Numerical modeling on each case (embankment or vegetation only) can be found in many references for instances see [1], [2] for the vegetation modeling and [3], [4] for the embankment modelling. In the work of Adytia et al. in [1], the model is based on shallow water equations with Manning friction. The model was solved numerically using staggered conservative scheme. Further, the shallow water model was also used to describe overtopping over sea dike as described in [4]. Wave interaction with vegetation can be modelled also using Navier-Stokes type equations as described in [2]. In the reference, influence of vegetation is considered as friction. Several frictions such as Manning, Darcy, and laminar, were used in the reference to model the influence of vegetation.

Wave run up is also often observed in the problem. An accurate run up prediction becomes important thing in the reseacrh field and several authors focused their research in the area for instances see [5], [6], [7], and [8]. Different model and numerical method are used to solve the problem. In the field of wave run up, data of laboratory experiment of Synolakis as described in 
detail in [6] are often used as a benchamrk test. The experimental data for several time $t$ are available online in [9].

According to our knowledge, numerical study in modelling the merged case is still few especially using staggered conservative scheme and shallow water equations. Therefore, in this study, we model the each case as well as the merged case using shallow water equations. Further, we solve the equations numerically using staggered conservative scheme. Purpose of this study is to develop model for investigating the reduction of wave run up due to existence of embankment and vegetation. To validate our numerical code in describing the wave run up, comparison between our numerical results and the data of Synolakis is carried out.

Outline of the article is as follows. Elaboration of numerical model including the governing equations and staggered conservative scheme will be given in Section 2. In Section 3, results and discussions of our numerical experiment will be presented. The section consists of three subsection; validation of the numerical code, run up reduction due to vegetation, and run up reduction due to embankment merged with vegetation. This article will be closed with some conclusions that will be given in Section 4.

\section{Numerical model}

In this section, elaboration of the numerical model (governing equations and the staggered conservative scheme) will be given.

\subsection{Governing equations}

Propagation of waves can be modelled using Shallow Water Equations (SWE). Consider the following equations

$$
\begin{aligned}
& h_{t}+(h u)_{x}=0 \\
& u_{t}+u u_{x}+g h_{x}=0
\end{aligned}
$$

Note that Equation (1) is derived from the mass conservation and Equation (2) comes from the momentum conservation. Further, notation $h(x, t)$ denotes the water level in which $h(x, t)=\eta(x, t)+d(x)$ where $\eta(x, t)$ and $d(x)$ represent the surface elevation and the seabed, respectively. Notation $u(x, t)$ is the depth averaged horizontal velocity. Whereas notation $t, x$, and $g$ denote time, position, and gravitational force, respectively.

In this article, the vegetation is considered as a friction term by adding Manning friction to the governing equations directly. Following Aditya in [1] and Gunawan [10], the Manning friction $S_{f}$ is given in Equation (3). Influence of the shoreline is cosidered in the bottom topography $d(x)$.

$$
S_{f}=\mu^{2} u|u| / h^{4 / 3}
$$

Notation $\mu$ in the Equation (3) represents Manning's roughness coefficient. 


\subsection{Staggered conservative scheme}

Here the staggered conservative scheme is used to solve the governing equations. The explanation of the method is described in detail in [11], [12], and [13]. In the method, the computational domain (time and space) is discretized into finite numbers of grids (cells) with constant length. The time interval $[0, T]$ is divided into a finite number of cells $N_{t}$ with length $\Delta t$. Whereas the spatial domain $\Omega=[0, L]$ is discretized into a finite number of grids $N_{x}$ with length $\Delta x$. Further, the water level is defined in the full grid and the velocity is defined in half grid. See Figure 1 as an illustration.

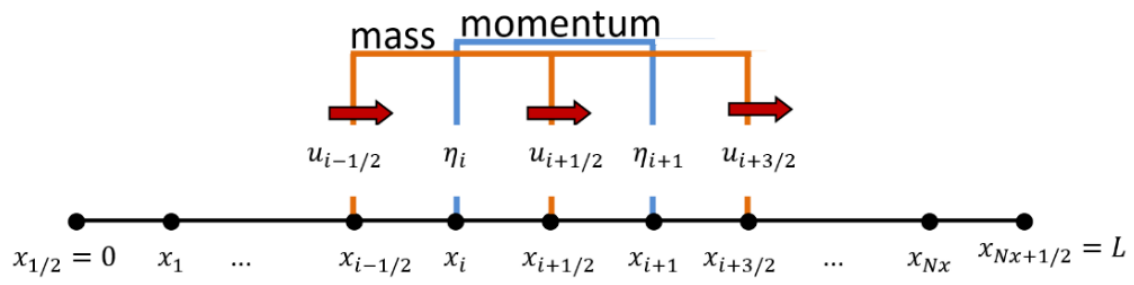

Fig. 1. Illustration of the staggered scheme discretization.

The discretizations and definitions lead to the following equation

$$
h_{i}^{n+1}=h_{i}^{n}-\left(\Delta t /_{\Delta x}\right)\left(h_{i+1 / 2}^{n} u_{i+1 / 2}^{n}-h_{i-1 / 2}^{n} u_{i-1 / 2}^{n}\right)
$$

Since the water level, $h$ is defined in the full grid so values of $h_{i+1 / 2}^{n}$ and $h_{i-1 / 2}^{n}$ do not exist. Here approximation of the missing values is needed. To solve the problem, the first-order upwind scheme is used. The upwind scheme can be written in the Equation (5).

$$
h_{i+1 / 2}=\left\{\begin{array}{cl}
h_{i} & \text { if } u_{i+1 / 2} \geq 0 \\
h_{i+1} & \text { if } u_{i+1 / 2}<0
\end{array}\right.
$$

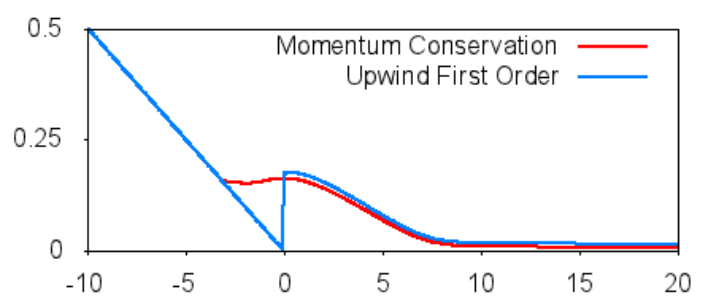

Fig. 2. Illustration of the non-linear approach.

It is important to choose the right scheme to approximate the non-linear term $u u_{x}$ in Equation (2). The choice is needed in order to describe run-up phenomena correctly (see Figure 2 as an illustration). In this article, an approximation based on momentum conservation is chosen instead of the first-order upwind scheme. Therefore, we modify the nonlinear term $u u_{x}$ as follows 


$$
u^{\partial u} / \partial x=(1 / h)\left(\partial\left(h u^{2}\right) / \partial x-u^{\partial(h u) / \partial x}\right)=(1 / h)\left(\partial(q u) / \partial x-u^{\partial q} / \partial x\right)
$$

Applying the staggered method to Equation (2) leads to the following staggered discretization

$u_{i+1 / 2}^{n+1}=u_{i+1 / 2}^{n}-\left(\Delta t /\left(\Delta x H_{i+1 / 2}^{n}\right)\right)\left(Q_{i+1}^{n} u_{i+1}^{n}-Q_{i}^{n} u_{i}^{n}-u_{i+1 / 2}^{n}\left(Q_{i+1}^{n}-Q_{i}^{n}\right)\right)$

where

$H_{i+1 / 2}^{n}=\left(h_{i+1}^{n}+h_{i}^{n}\right) / 2, \quad Q_{i}^{n}=\left(q_{i+1 / 2}^{n}+q_{i-1 / 2}^{n}\right) / 2, \quad q_{i+1 / 2}^{n}=h_{i+1 / 2}^{n} u_{i+1 / 2}^{n}$

Due to the definition of the velocity $u$ that is defined in the half grid so values of $u_{i+1}^{n}$ and $u_{i}^{n}$ do not exist too. Here the first-order upwind scheme is also used

$$
u_{i}= \begin{cases}u_{i-1 / 2} & \text { if } Q_{i} \geq 0 \\ u_{i+1 / 2} & \text { if } Q_{i}<0\end{cases}
$$

Following another research in [1], discretization of the Manning friction term (see Equation (3) is written as

$$
S_{f_{i+1 / 2}^{n}}^{n}=\mu^{2} u_{i+1 / 2}^{n+1}\left|u_{i+1 / 2}^{n}\right| /\left(h_{i+1 / 2}^{n+1}\right)^{4 / 3}
$$

\section{Numerical results and discussions}

Detail of numerical experiments will be elaborated in this section. Three kinds of numerical simulations are conducted. The first one is conducted to show the performance of our numerical code in describing run upon a simple beach. Here, the numerical results are compared with the experimental data of Synolakis provided in [9]. The second simulation is carried out to see the reduction of run-up on the simple beach due to the existence of vegetation. Whereas the last numerical experiment is provided to investigate the influence of vegetation and embankment on the reduction of wave run-up.

\subsection{Validation of the numerical code}

To validate our numerical code in wave propagation and wave run-up on the simple beach, comparison with data of laboratory experiments of Synolakis is conducted. In this numerical simulation, the set up of initial wave, flow velocity, and bathymetry of the beach are chosen as described in [6] and [8]. Further, in the simulation, ratio of amplitude $H$ and constant depth $d$ is taken as $\frac{H}{d}=0.0185$. Values of the other parameters are time step $\Delta t=0.000845$, width of the grid $\Delta \mathrm{x}=0.02777$, and computational domain $\Omega=[-10,70]$. The results of the comparison are presented in Figure 3 . 
Figure 3 shows the comparison of results of the numerical simulation and data of the laboratory experiment of Synolakis. Initial data of the simulation are given in Figure 3 (a). The wave propagates (Figure 3 (b)) to the shoreline and reaches the shoreline (Figure $3(\mathbf{c})$ ). Figure 3 (b) and Figure 3 (c) show that the numerical results are in good agreement with the experimental data in describing wave propagation until the shoreline. Figure 3 (d) - Figure 3 (f) shows that the numerical solution is comparable with the data in the depiction of the wave run-up.
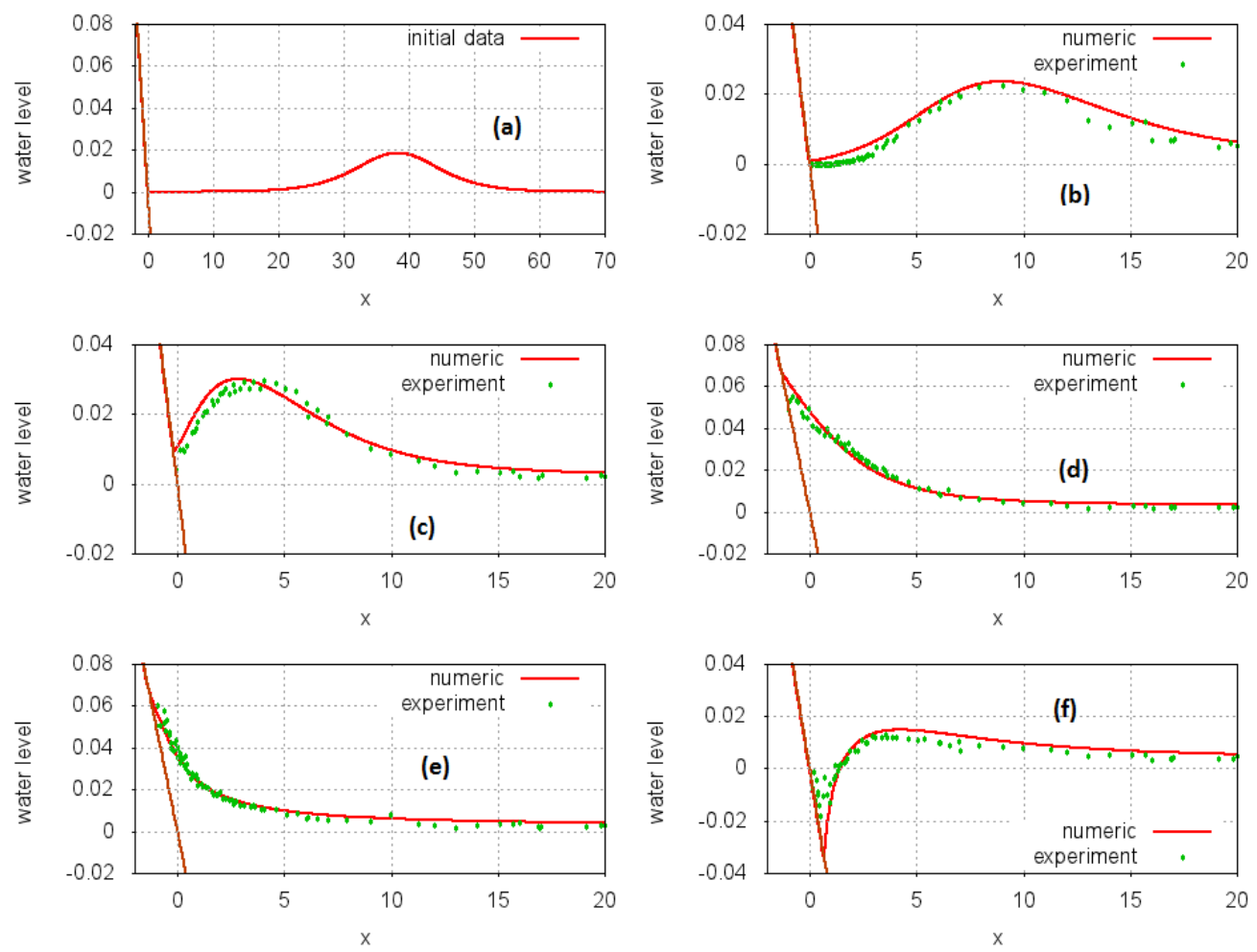

Fig. 3. Comparison between the numerical result (line) and experimental data of Synolakis [9] (dotted line) at time $\mathrm{t}=0 \mathrm{t}_{0}(\boldsymbol{a}), 30 \mathrm{t}_{0}(\boldsymbol{b}), 40 \mathrm{t}_{0}(\boldsymbol{c}), 50 \mathrm{t}_{0}(\boldsymbol{d}), 60 \mathrm{t}_{0}(\boldsymbol{e}), 70 \mathrm{t}_{0}(\boldsymbol{f})$ where $t_{0}=\sqrt{\mathrm{g} / \mathrm{d}}$.

\subsection{Run up reduction due to the vegetation}

The simulation is conducted to see the influence of vegetation on the reduction of wave run-up. Note that, in this simulation values of all parameters, initial wave, initial flow velocity, and bathymetry are set to be the same as the previous simulation. The vegetation is placed in area $0 \leq x \leq 2$. In the program, the vegetation is seen as Manning friction with a value of constant $\mu_{f}=0.04$. The constant value is arbitrary chosen. The results of the simulation are given in Figure 4.

Figure 4 shows a comparison of numerical results of vegetation case in which the vegetation exists and normal cases in which there is not any vegetation or embankment. Figure 4 (a) shows that the vegetation does not give influence yet. The influence of the vegetation is 
starting to exist in Figure 4 (b). In Figure 4 (c), the value of the run-up of the vegetation case is lower than the normal case. The run-up height of the normal case and the vegetation case are 0.063963 and 0.05323 , respectively. It means that there is a reduction $16.78 \%$ of run-up height due to the existence of the vegetation.
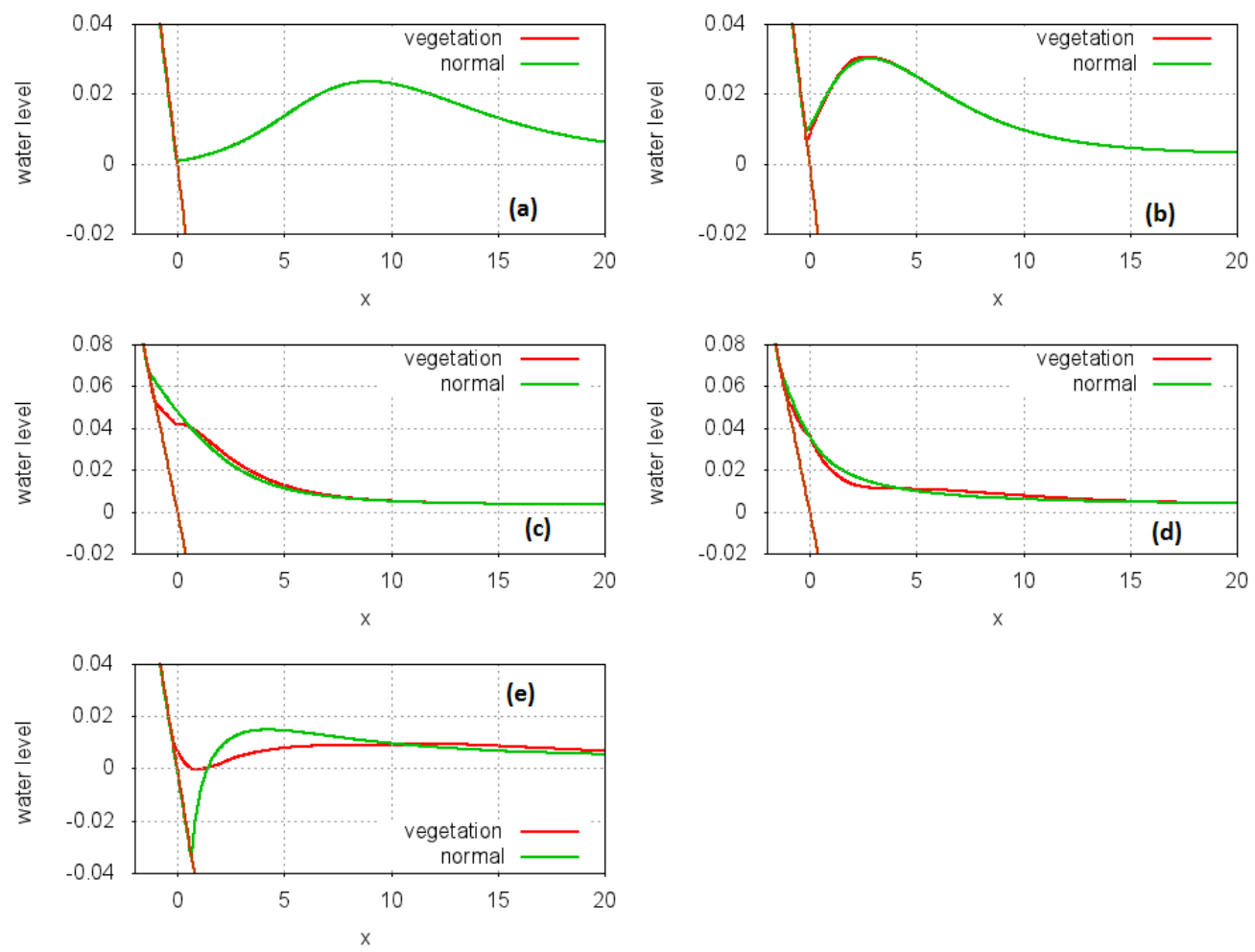

Fig. 4. Comparison between the numerical result of vegetation case (red line) and normal case (green line) at time $\mathrm{t}=30 \mathrm{t}_{0}(\boldsymbol{a}), 40 \mathrm{t}_{0}(\boldsymbol{b}), 50 \mathrm{t}_{0}(\boldsymbol{c}), 60 \mathrm{t}_{0}(\boldsymbol{d}), 70 \mathrm{t}_{0}(\boldsymbol{e})$ where $t_{0}=\sqrt{g / d}$.

\subsection{Run up reduction due to the vegetation and embankment}

The numerical experiment is conducted to see the influence of vegetation merged with an embankment in describing wave propagation and wave run-up phenomena. Set up of the simulation is the same as the previous simulation. Further, the embankment is added to the topography using equation

$$
d(x)=-0.19 x-0.14 x^{2}-0.036 x^{3},
$$

where $-2 \leq x \leq 0$. In the merged case, both vegetation and embankment are added to the simulation. The location and constant value of Manning friction are the same as the previous simulation. Whereas the embankment is created with the Equation (2). The results of the simulation are given in Figure 5.

Figure 5 presented a comparison between the results of the embankment case and the merged case. It is clearly seen in Figure 5 (c) that the results of the cases are different. Run up 
of the merged case is lower than the embankment case. Run-up the height of the merged case and the embankment case are 0.070763 and 0.075111 , respectively. Therefore there is a reduction of run-up height about $5.79 \%$ respect to the embankment case. In Figure 5 (d) and Figure 5 (e), the amplitude of the reflection wave of the merged case is lower.
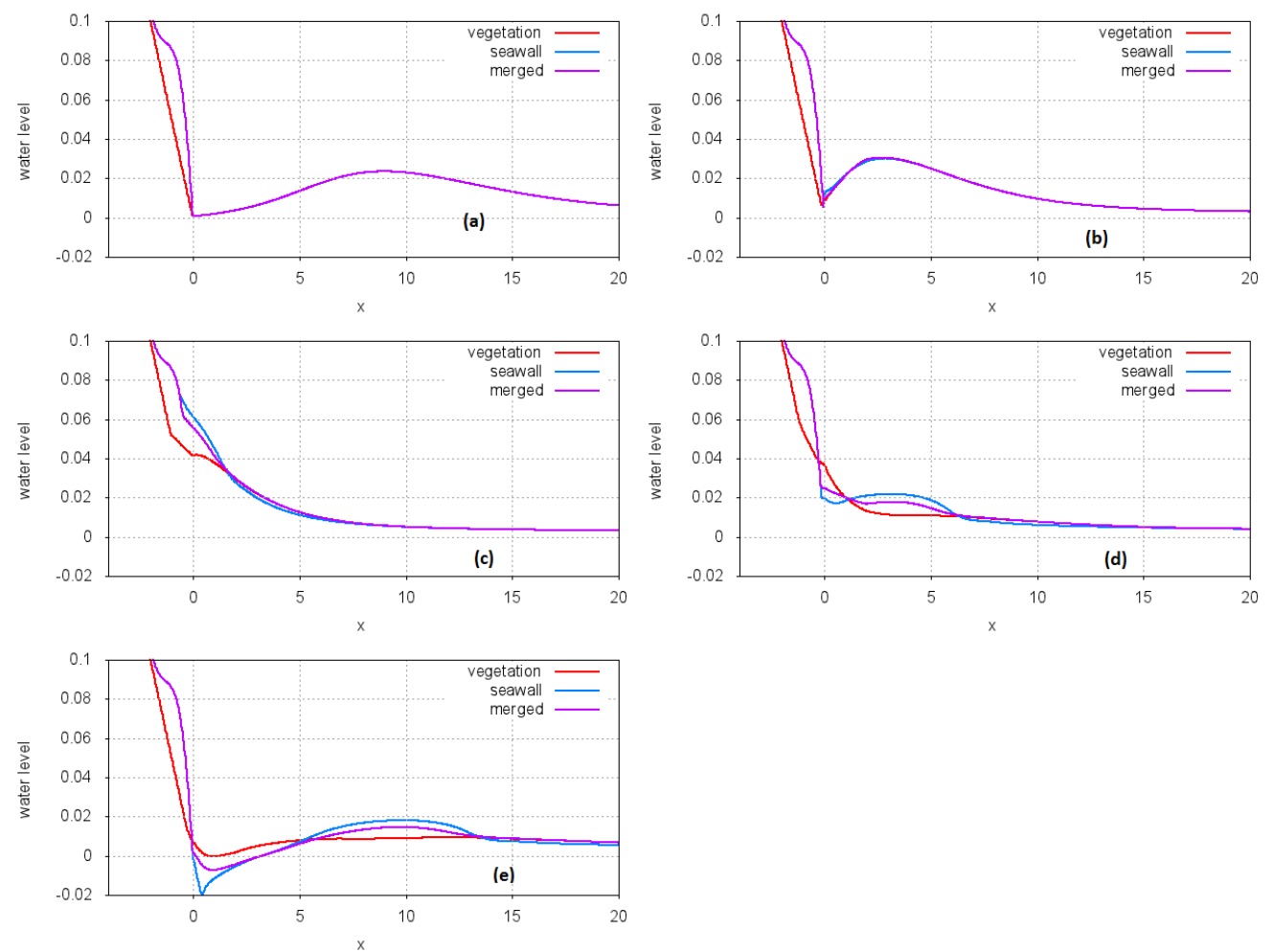

Fig. 5. Comparison between numerical result of vegetation case (reed line), embankment case (blue line), and merged case (purple line) at time $\mathrm{t}=30 \mathrm{t}_{0}(\boldsymbol{a}), 40 \mathrm{t}_{0}(\boldsymbol{b}), 50 \mathrm{t}_{0}(\boldsymbol{c}), 60 \mathrm{t}_{0}(\boldsymbol{d}), 70 \mathrm{t}_{0}(\boldsymbol{e})$ where

$$
t_{0}=\sqrt{g / d}
$$

\section{Conclusions}

The numerical model for investigating the effectiveness of embankment and vegetation on the reduction of the wave run-up has been elaborated. The model based on shallow water equations solved numerically using the staggered conservative scheme. The Manning friction is used to describe the influence of vegetation. To validate our numerical code in describing wave run-up phenomena, comparison with experimental data of Synolakis has been conducted. The results show that numerical results are in good agreement with the data. From the second numerical experiment, the existence of the vegetation can reduce $16.78 \%$ of run-up height respect to the normal case. Whereas from the third simulation, the existence of the vegetation and embankment can reduce $5.79 \%$ of run-up height respect to the embankment case. 
Acknowledgments. The authors acknowledge Riset Internal Politeknik Negeri Indramayu for funding this research.

\section{References}

[1] Adytia, D., Husrin, S., \& Latifah, A. L. (2019).: Dissipation of Solitary Wave Due To Mangrove Forest: A Numerical Study by Using Non-Dispersive Wave Model. Indonesian Journal of Marine Sciences/Ilmu Kelautan, 24(1).

[2] Gunawan, P. H. (2016, February).: Simulation of wave mitigation by coastal vegetation using smoothed particle hydrodynamics method. In Journal of Physics: Conference Series (Vol. 693, No. 1, p. 012013). IOP Publishing.

[3] Altomare, C., Suzuki, T., Chen, X., Verwaest, T., \& Kortenhaus, A. (2016).: Wave overtopping of sea dikes with very shallow foreshores. Coastal Engineering, 116, 236-257.

[4] Suzuki, T., Altomare, C., Veale, W., Verwaest, T., Trouw, K., Troch, P., \& Zijlema, M. (2017). Efficient and robust wave overtopping estimation for impermeable coastal structures in shallow foreshores using SWASH. Coastal Engineering, 122, 108-123.

[5] Fiedler, J. W., Smit, P. B., Brodie, K. L., McNinch, J., \& Guza, R. T. (2018).: Numerical modeling of wave runup on steep and mildly sloping natural beaches. Coastal Engineering, 131, 106-113.

[6] Synolakis, Costas Emmanuel.: The runup of solitary waves. Journal of Fluid Mechanics. Vol. 185, pp 523-545 (1987)

[7] Adytia, D., Pudjaprasetya, S. R., \& Tarwidi, D. (2019).: Modeling of wave run-up by using staggered grid scheme implementation in 1D Boussinesq model. Computational Geosciences, 1-19.

[8] Iryanto, \& Pudjaprasetya, S. R. (2017).: A Coupled Model for Wave Run-up Simulation. East Asian Journal on Applied Mathematics, 7(4), 728-740.

[9] The NOAA Center for Tsunami Research.: Solitary wave on a canonical beach, http://nctr.pmel.noaa.gov/benchmark/Laboratory/Laboratory_CanonicalBathymetry/index.html, accessed on 10 September 2019.

[10] Gunawan, P. H., \& Lhébrard, X. (2015).: Hydrostatic relaxation scheme for the 1D shallow waterExner equations in bedload transport. Computers \& Fluids, 121, 44-50.

[11] S. R. Pudjaprasetya and I. Magdalena.: "Momentum conservative schemes for shallow water flows," East Asian Journal on Applied Mathematics, vol. 4, pp. 152-165, 0052014.

[12] G. S. Stelling and S. A. Duinmeijer.: "A staggered conservative scheme for every froude number in rapidly varied shallow water flows," International Journal for Numerical Methods in Fluids, vol. 43, no. 12, pp. 1329-1354, 2003.

[13] G. Stelling and M. Zijlema.: "An accurate and efficient finite-difference algorithm for nonhydrostatic free-surface flow with application to wave propagation," International Journal for Numerical Methods in Fluids, vol. 43, no. 1, pp. 1-23, 2003. 